University of Nebraska - Lincoln

DigitalCommons@University of Nebraska - Lincoln

Faculty Publications in Computer \& Electronics Electrical \& Computer Engineering, Department Engineering (to 2015)

2011

\title{
Battery Capacity Footprinting and Optimization Analysis for Wireless Multimedia Communication
}

Jianxin Sun

University of Nebraska-Lincoln, jianxin.sun@huskers.unl.edu

Dalei Wu

University of Nebraska-Lincoln, dalei-wu@utc.edu

Song $\mathrm{Ci}$

University of Nebraska-Lincoln, sci@engr.unl.edu

Follow this and additional works at: https://digitalcommons.unl.edu/computerelectronicfacpub

Part of the Computer Engineering Commons

Sun, Jianxin; Wu, Dalei; and Song Ci, "Battery Capacity Footprinting and Optimization Analysis for Wireless Multimedia Communication" (2011). Faculty Publications in Computer \& Electronics Engineering (to 2015). 87.

https://digitalcommons.unl.edu/computerelectronicfacpub/87

This Article is brought to you for free and open access by the Electrical \& Computer Engineering, Department of at DigitalCommons@University of Nebraska - Lincoln. It has been accepted for inclusion in Faculty Publications in Computer \& Electronics Engineering (to 2015) by an authorized administrator of DigitalCommons@University of Nebraska - Lincoln. 


\title{
Battery Capacity Footprinting and Optimization Analysis for Wireless Multimedia Communication
}

\author{
Jianxin Sun ${ }^{1}$, Dalei $\mathrm{Wu}^{1}$, Song $\mathrm{Ci}^{1,2}$ \\ ${ }^{1}$ Department of Computer and Electronics Engineering, University of Nebraska-Lincoln, NE 68182, USA \\ Email: \{jianxin.sun,dwu\}@ huskers.unl.edu, sci@engr.unl.edu \\ ${ }^{2}$ High Performance Network Lab, Chinese Academy of Sciences, Beijing 100190, China
}

\begin{abstract}
Micro-electronic industry has been boosting the capability of wireless mobile devices on full-scale. However, battery, as the only power source of most mobile devices, is experiencing a relatively slow development. Therefore, how to optimally utilize the limited battery energy on mobile devices under a predefined performance requirement becomes a critical issue. On the other hand, it is still unclear that how the battery capacity consumption is allocated on different working pattern of a specific video codec under various tempo-spatial scales and parameters, which has posed a design challenge on power management on multimedia communication system. Furthermore, an optimization method is needed to be proposed and experimentally tested to achieve the tradeoff between the computational complexity and the distortion of multimedia delivery in order to discover the relationship and interaction between computational parameters of multimedia communication and battery capacity consumption. In this paper, we set up a measurement system to reveal the battery capacity consumption behavior and its footprinting in a video delivery system using $\mathbf{H . 2 6 4}$ codec. A systematic optimization framework which jointly considers the coding parameters and transmission parameters is proposed to achieve the tradeoff between battery capacity consumption and quality of services $(Q 0 S)$. Experimental results indicate insight of relationship between multimedia communication parameters and battery capacity consumption under a desired distortion requirement which provides a guideline of optimization for applicable multimedia system.
\end{abstract}

\section{INTRODUCTION}

Mobile multimedia on portable wireless devices has been greatly enhanced by the network infrastructure upgrade and development in video communication technology over wireless network. Although wireless video communication is highly desirable, a primary limitation in wireless systems is the basic design architecture that most mobile devices are typically powered by batteries with limited capacity. Many researches have been engaged in the improvement of battery performance, but, due to the technical difficulties and financial issue, it is still counterproductive to rely on the battery improvement to narrow the gap between high energy consumption of multimedia processing and the limited battery capacity. From the perspective of battery-aware design and power management, how to wisely perform the energy allocation is a critical issue to guarantee a required service quality. In literature, complexity control parameters in those steps of a generic video encoder was investigated in [1]. However, video transmission

This work was partially supported by NSF under Grant No. CCF-0830493 and ECCS-0801736. was not considered in that work. Although energy efficiency of both video coding and transmission were studied in [2] [3] [4], only power was considered by defining the mathematic relationship between coding parameters and power consumption instead of battery capacity consumption which is significant in revealing the battery working manner in multimedia delivery. Currently, no dedicated analytical framework or experimental analysis on battery capacity consumption under the context of H.264 codec has been performed. In this paper, we set up a battery capacity measurement testbed for multimedia delivery application based on H.264 codec, which will footprint the battery capacity consumption in video coding and transmission. Based on the profiles from measurements, complexity control parameters in both coding and transmission processes will be jointly selected by applying proposed optimization algorithm in order to minimize the battery capacity consumption under a certain constrain of expected received end-to-end distortion.

This paper is organized as follows. Section II presents the formulation of the problem to solve. In Section III and IV present the methods battery capacity consumption profiling and expected end-to-end distortion profiling. A systematic optimization framework is proposed in Section V. Section VI presents the experimental results. Concluding remarks are given in Section VII.

\section{Problem Statement}

\section{A. Capacity Consumption on Video Coding}

The major modules in a typical video encoding system include motion estimation (ME) and compensation, DCT, quantization, entropy encoding of the quantized DCT coefficients, inverse quantization, inverse DCT, picture reconstruction, and interpolation. Computational complexity and power consumption of these modules have been evaluated in [1], [5], [6]. It has been shown that, for each module in video coding process, a set of system parameters of video codec can be selected to control the computational complexity of that module. Let $\Gamma=\left[\gamma_{1}, \gamma_{2}, \cdots, \gamma_{I}\right]$ be the set of control parameters to control the computational complexity of those modules in video coding process. Therefore, the battery capacity consumption of the underlying microprocessor to compress a video clip, denoted by $C_{e}$, is a function of $\Lambda$, denoted by

$$
C_{e}=C_{e}(\Gamma)=C_{e}\left(\gamma_{1}, \gamma_{2}, \cdots, \gamma_{I}\right)
$$




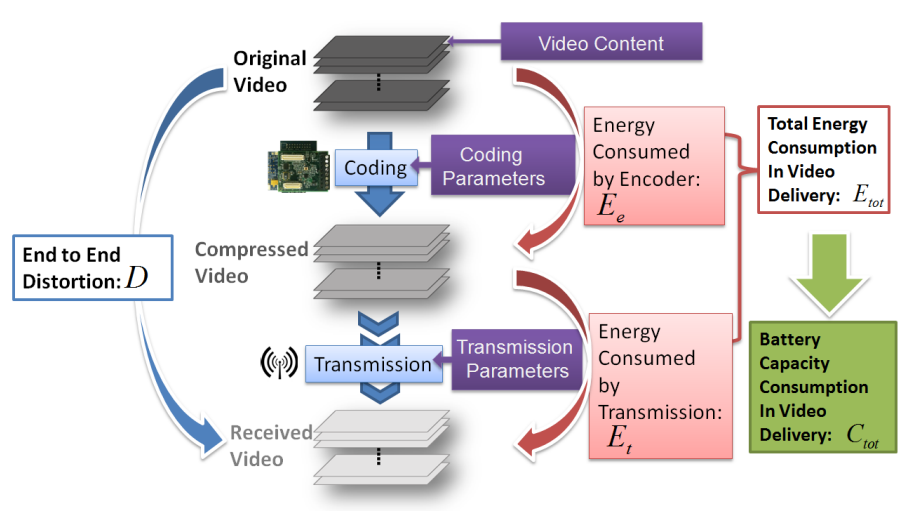

Fig. 1. Wireless video delivery scheme

\section{B. Capacity Consumption on Video Transmission}

To analyze the battery capacity consumption on video transmission, we need to consider both the transmission scheme and the power control technology adopted by the transmitter on mobile devices. In general, the capacity $C_{t}$ consumed in transmitting video data not only depends on wireless channel conditions, such as the instantaneous channel fading factor and channel noise power density, but also on transmission parameters, such as frequency bandwidth, desirable packet error rate (PER), and modulation and coding schemes. Without loss of generality, let $\Theta=\left[\theta_{1}, \theta_{2}, \cdots, \theta_{J}\right]$ be the set of parameters affecting or controlling the transmission energy level. Therefore, the capacity used in transmission can be represented by

$$
C_{t}=C_{t}(\Theta)=C_{t}\left(\theta_{1}, \theta_{2}, \cdots, \theta_{J}\right) .
$$

\section{Problem Formulation}

Figure 1 shows the key steps to accomplish video delivery. Video coding and transmission are two main processes, and each one of them will consume capacity from battery. On the other hand, when the whole video clip is delivered, A distortion will be generated at the receiving terminal. In order to guarantee the service quality, this end-to-end distortion has to be kept lower than a required threshold. Let $D$ be the expected video distortion, and it depends on the video processing parameters $\Gamma$ and the transmission parameters $\Theta$.

Based on Eqs. (1) and (2), the total battery capacity consumption required to deliver a video clip is

$$
C_{t o t}=C_{e}+C_{t} .
$$

Since different load levels result in different battery operating time, the objective of the proposed framework is to determine the optimal values of the parameters $\{\Gamma, \Theta\}$ for a given sequence of video frames to minimize the battery capacity consumption under the constraint on distortion $D_{\max }$, which can be formulated as

$$
\begin{aligned}
\min _{\{\Gamma, \Theta\}} C_{t o t}(\Gamma, \Theta) \\
\text { s.t. : } D(\Gamma, \Theta) \leq D_{\max } .
\end{aligned}
$$

\section{BAtTery CAPACity Consumption Profiling}

\section{A. Battery Capacity Consumption on encoding}

Experiment has showed that it is reasonable to adopt the quantization step size $q$, which is the key parameter in PRECODING [1] process, as one of the optimal complexity control parameters. On the other hand, from the perspective of inter coding, maximal search range $r$ and number of previous frames used for inter motion search (number of reference frames $n$ ) also play very important roles in controlling the computational complexity during coding process. As a result, we select these three coding parameters as the control parameters in coding.

In this work, we have established a testbed with the considerations of high-resolution battery measurements. The hardware measurement system is Imote2 wireless sensor node connected with Arbin Battery Testing System. The H.264 video codec runs on a Linux-based Imote2 wireless sensor node with a PXA271 XScale processor. In order to get a more standard result, we set the CPU as a constant frequency(the frequency of Performance Mode of Imote2), that means no dynamic voltage or frequency scaling is applied. A PC terminal receives and records two kinds of data from the Arbin battery testing equipment: the current value and the battery capacity consumption. Meanwhile, during the coding process, Imote 2 records and transmits the related key information to the PC terminal, such as coded frame bits $F_{k}$ and frame coding time $t_{k}$ (the time elapse to code the $k$ th frame). H.264 codec is stored in the flash memory of Imote 2 under different codec configuration. The three coding control parameters, $q, r$ and $n$, can be adjusted by modifying the baseline configuration file every time before running the codec. During the video coding process, when compression of the $k t h$ video frame is finished, the codec will output the processing time $t_{k}$ of that frame and the size $F_{k}$ of coded frame bits. Once the control parameters are fixed, we can establish a relationship between $t_{k}$ and the total battery capacity usage started from the beginning of the test at the time point $t_{0}$ to $t_{k}$. In general, the coding battery capacity consumption profile of each frame is a set of data based on the three coding control parameters and content of the $k$ th frame, and it can be denoted as

$$
C_{e}^{k}=C_{k}-C_{k-1}=C_{e}(q, r, n, k) .
$$

\section{B. Battery Capacity Consumption on Transmission}

The energy used to transmit a frame depends on the number of bits of coded frame and the current wireless channel capacity. Usually, the number of bits per coded frame is decided by the video content and the three coding parameters adopted in coding that frame. The transmission rate of a wireless channel depends on the current channel quality and the adaptive modulation and coding (AMC) scheme. Different AMC schemes will result in different transmission rates and spectrum efficiency. Table I shows the sigmoid parameters $(\lambda, \delta)$ for the 8 AMC schemes in modeling packet transmissions over an 802.11a WLAN network. Let $W$ be the wireless 


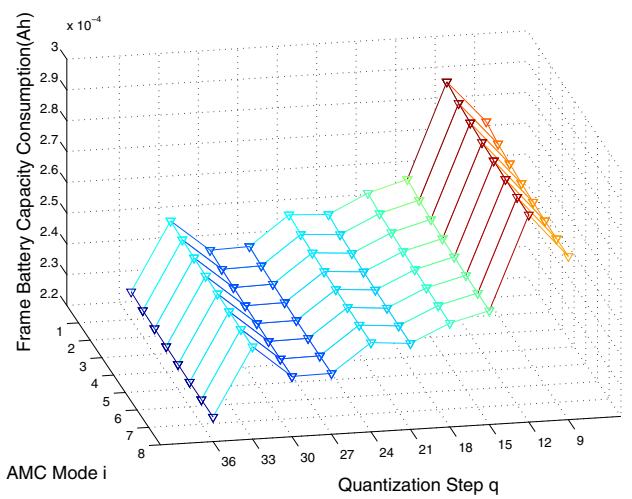

Fig. 2. Battery capacity consumption profile of the $25 t h$ frame under control parameters of $q$ and $i(s=4, n=1)$

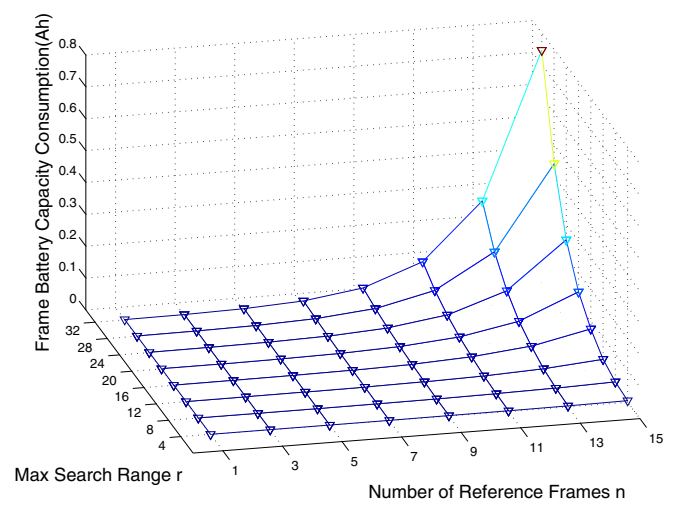

Fig. 3. Battery capacity consumption profile of the $25 t h$ frame under control parameters of $s$ and $r(q=21, i=1)$

channel bandwidth, and $K_{i}$ be the transmission rate of AMC scheme $i$ which can be calculated from table I by applying the method introduced in [7]. Then, the transmission rate of the $i$ th AMC scheme is $K_{i} \cdot W$. Denote $F_{k}$ to represent the number of coded bits of the $k$ th frame and it is determined by the corresponding measurement. let $P$ be the transmission power. The battery capacity consumption of one frame can be derived if the operating voltage of hardware platform $V$ is known, therefore,

$$
C_{t}^{k}=\frac{\sum_{k=1}^{n} P \cdot \frac{F_{k}}{K_{i} \cdot W}}{V} .
$$

Because $F_{k}$ depends on the coding process, so it is determined by the previous three coding control parameters as well, In general, the battery capacity consumed on delivering the $k$ th frame is a function of the frame number, the AMC scheme $i$, and coding control parameters, which is

$$
C_{t}^{k}=C_{t}(q, r, n, i, k)
$$

The total battery capacity consumption in delivering one frame is the sum of the capacity usage in both coding process and transmission process.

$$
C_{t o t}^{k}=C_{e}^{k}+C_{t}^{k}
$$

TABLE I

PACKET ERROR PROBABILITY OF DIFFERENT AMC SCHEMES

\begin{tabular}{|c|c|c|c|c|}
\hline Mod Scheme & $\begin{array}{c}\delta \\
(d B)\end{array}$ & $\begin{array}{c}\lambda \\
\left(d B^{-1}\right)\end{array}$ & $\begin{array}{c}\text { CodeRate } \\
(\text { bits } / \text { symbol })\end{array}$ & $\begin{array}{c}A M C \\
(i)\end{array}$ \\
\hline BPSK & 2.3 & 0.640 & 0.5 & 1 \\
\hline BPSK & 6.1 & 0.417 & 0.75 & 2 \\
\hline QPSK & 5.3 & 0.461 & 1 & 3 \\
\hline QPSK & 9.3 & 0.444 & 1.5 & 4 \\
\hline 16-QAM & 10.9 & 0.375 & 2 & 5 \\
\hline 16-QAM & 15.1 & 0.352 & 3 & 6 \\
\hline 64-QAM & 18.2 & 0.625 & 4 & 7 \\
\hline 64-QAM & 21.2 & 0.419 & 4.5 & 8 \\
\hline
\end{tabular}

Figure 2 and 3 show the battery capacity consumption profile of the 25th frame based on control parameter vector ( $q$, $i)$ and $(r, n)$. Figure 2 shows that AMC scheme does not have a obvious contribution as quantization step does on battery capacity consumption. From figure 3 , we notice that the maximal search range can change the battery capacity consumption more efficiently than number of reference frames.

\section{EXPECTED END-TO-ENd Distortion ProfiLING}

In this work, the received video quality is evaluated as the expected end-to-end distortion by using the ROPE (Recursive Optimal per-Pixel Estimate) method. Therefore, given the dependency introduced by the error concealment scheme, the expected distortion of slice/packet $\pi_{i}$ can be calculated as

$$
\begin{array}{r}
E\left[D_{i}\right]=\left(1-p_{i}\right) E\left[D_{i}^{R}\right]+p_{i}\left(1-p_{i-1}\right) E\left[D_{i}^{L R}\right] \\
+p_{i} P i-1 E\left[D_{i}^{L L}\right],
\end{array}
$$

where $p_{i}$ is the loss probability of packet $\pi_{i}, E\left[D_{i}^{R}\right]$ is the expected distortion of packet $\pi_{i}$ if received, and $E\left[D_{i}^{L R}\right]$ and $E\left[D_{i}^{L L}\right]$ are respectively the expected distortion of the lost packet $\pi_{i}$ after concealment when packet $\pi_{i-1}$ is received or lost. The expected distortion of the whole video frame, denoted by $E[D]$, can be written as

$$
E[D]=\sum_{i=1}^{I} E\left[D_{i}\right] .
$$

Generally speaking, multiple modulation and coding schemes are available to achieve a good tradeoff between the transmission rate and the packet error rate. The error probability of a packet of $L$ bytes, under a given AMC scheme $i$, is a function of the bit error probability $p_{b, i}$, which can be express as $p_{e, i}(L)=1-\left(1-p_{b, i}\right)^{8 L}$. Moreover, $p_{b, i}$ can be approximated with sigmoid functions in [8] and [9] as

$$
p_{b, i}(L)=\frac{1}{1+e^{\lambda(x-\delta)}},
$$

where $x$ is the Signal-to-Interference-Noise-Ratio (SINR). From this table I and (11), it can be observed that $p_{e, i}$ depends on the AMC scheme $i$, and so does the overall distortion, since the end-to-end distortion is the function of $p_{e, i}$. Once the packet error probability is calculated, the expected endto-end distortion will be calculated based on Eqs. (9) and (10). In other words, the set of coding control parameters 


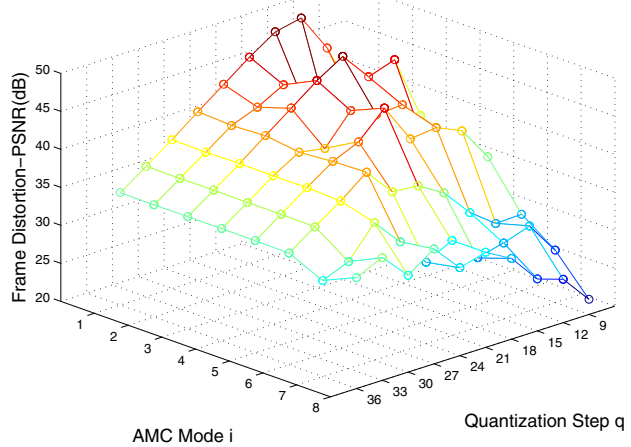

Fig. 4. Received end-to-end distortion profile of the $25 t h$ frame under control parameters of $q$ and $i(s=4, n=1)$

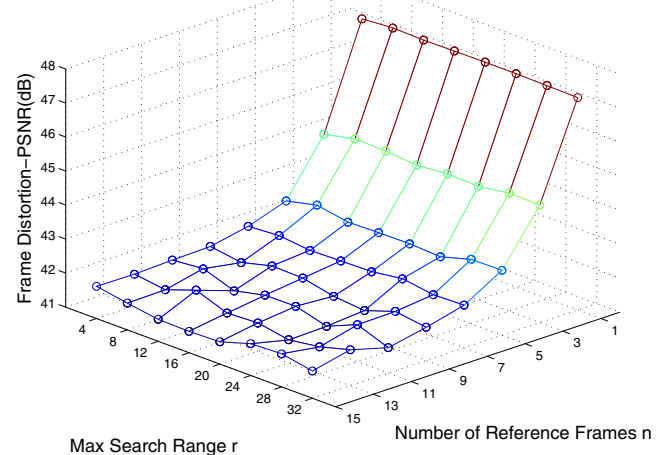

Fig. 5. Received end-to-end distortion profile of the 25th frame under control parameters of $s$ and $r(q=21, i=1)$

need to be considered as other effective system parameters to reduce the total video distortion. Based on the statistic evaluation that SINR dose not change frequently on a common communication scenario, we set $x$ as a reasonable constant in our later experiments. Therefore, the expected frame distortion associated with AMC scheme $i$ and coding control parameters can be denoted as

$$
E[D]_{k}=D(q, r, n, i, k) .
$$

Figures 4 and 5 show the received end-to-end distortion profile of the 25th frame based on control parameter vector $(q, i)$ and $(r, n)$. Figure 4 shows that AMC scheme and quantization step jointly affect the distortion of the one video frame. From figure 5, we notice that the distortion can be affected by adjusting the number of reference frames. Meanwhile, max search range does not have appreciable influence on distortion.

\section{OPTIMIZATION FRAMEWORK}

Since we have established the two profile on battery capacity consumption and received end-to-end distortion, and both of the profiles are based on three coding parameters and one transmission parameter. Coding parameters can be adjusted by setting configuration file of encoder. And in the streaming
TABLE II

ALGORITHM OF BATTERY CAPACITY OPTIMIZATION FRAMEWORK

For each frame $\mathrm{n}=1,2, \cdots$
Distortion profile $P_{d}$
Coding battery capacity consumption profile $P_{c}^{c}$
Transmission battery capacity consumption profile $P_{c}^{t}$
Battery capacity consumption profile $P_{c}=P_{c}^{c}+P_{c}^{t}$
For each Control Vector $(q, i, r, n) \in$ Possible Set $\Psi$
If $P_{d}(q, i, r, n)<D_{\max }$
Add this Control Vector into Selected Set $\Omega$
Next Control Vector $(q, i, r, n)$
For each Control Vector $(q, i, r, n) \in$ Selected Set $\Omega$
If Control Vector $(q, i, r, n)$ has the minimized $P_{c}$
$\quad$ Label this $(q, i, r, n)$ as the optimal Control Vector
Next Control Vector $(q, i, r, n)$
Next $\mathrm{n} \quad$

system, intermediate buffer between video coder and network subsystem takes charge of adjusting the transmission parameter at frame level. For simplicity, we establish a four dimension vector $(q, i, r, n)$ to stand for all the combination of possible control parameters. Let $Q$ be the total options of $Q P, I$ the total optional AMC schemes, $R$ the total optional max search range and $N$ the total optional number of reference frame. So every video frame has $Q \cdot I \cdot R \cdot N$ options of this vector, and all the options form a possible set $\Psi$.

Therefore, the task is to find the best control vector toward each frame to minimize the battery capacity consumed on delivering each frame under the constraint of expected received frame distortion. By applying our measurement hardware platform, we can derive received frame distortion profile and frame coding battery capacity consumption profile from the experiments of measurement. If we set the received frame distortion constraint as a certain level, every control vector result in the distortion smaller than this constraint is representing a qualified options and all of these qualified control vectors form a selected set $\Omega$. By referring to the battery capacity consumption profile of a given frame, we can select the best control vector which has the minimized battery capacity consumption while satisfies the received frame distortion constraint. The algorithm of this optimization framework is described in Table II.

\section{EXPERIMENTAL RESULT}

We have conducted experiments to evaluate the performance of the proposed framework. The testbed is in charge of monitoring and recording all the desired battery operation data. The first 50 frames of each video sequence is encoded with the H.264 codec (JVT reference software, JM 16.2 [10]). We chose the quantization step size $(q)$, max search range $(r)$, number of reference frames $(n)$ and AMC schemes $(i)$ as the tunable source coding and transmission parameters. The permissible QP $q$ values are $[9,12,15 \ldots, 36]$, the values of $r$ are $[4,8,12 \ldots, 42]$, and the values of $n$ are $[1,3,9 \ldots, 15]$. According to Table I, the permissible AMC schemes index $i$ are $[1,2,3, \ldots, 8]$. The optimization framework proposed in this paper was firstly tested by three experiments under 


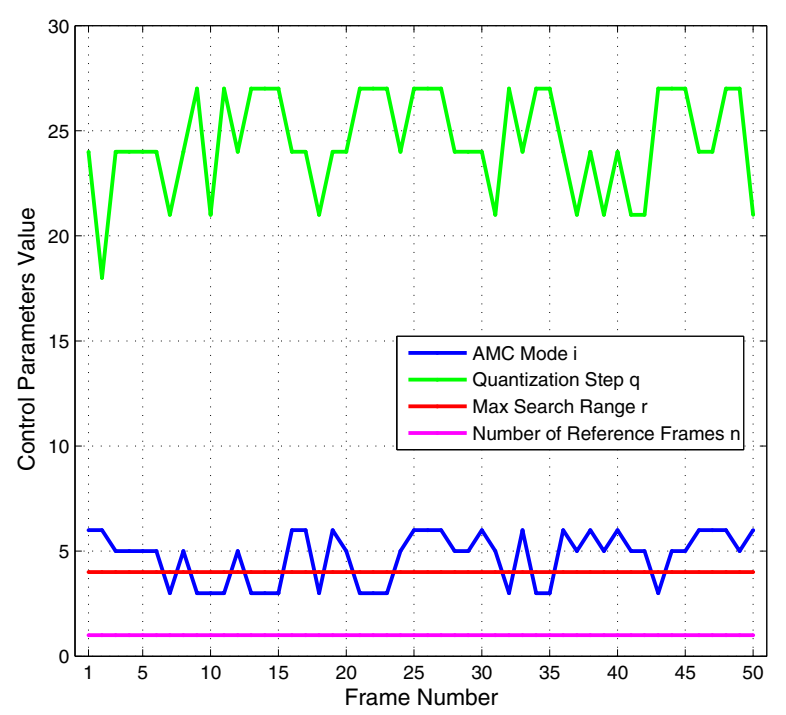

Fig. 6. Optimized control vectors corresponding to the first 50 frames under frame distortion constraints of $42 d B$ of PSNR

different distortion constraints. In the first experiment, we took the first 50 frames of the "Foreman" video clip and set the average frame distortion constraint at $36 d B$ of PSNR. And the other two were executed under the average frame distortion constraint of $42 d B$ and $47 d B$.

In the experiment of control vectors optimization corresponding to the first 50 frames of the tested video clip. Every vector which consists of 4 parameters represents one optimal control set according to frame number which satisfy the frame distortion constraint. All the optimal control vectors of these 50 frames have formed an optimal solution for this video clip. Figure 6 is showing changing of all the four optimal parameters of the first 50 frames of the tested video clip under the constraint of $42 d B$ of PSNR. we also observe that the gain on battery capacity consumption increases linearly with the total number of video frames and the optimization that involves more control parameters can result in better energy efficiency. Thus, the proposed optimization framework can save a considerable amount of battery capacity by adopting more control parameter in the optimization framework when it is applied to the cases of long-duration video deliveries.

According to the additional experiments we tested under the other values of PSNR constraints, we observed that different parameters choices in optimal results follow a predictable changing pattern: The overall value of quantization step tends to decrease as required PSNR increases, but the detail value for each frame is slightly effected by the current frame content. Max search range and number of reference frames grows according to the increase of PSNR requirement, but former is more effective then the later in controlling the battery capacity consumption. Transmission parameter AMC tends to decrease as the required PSNR increases, but it also slightly fluctuates due to different frame content. Without losing generality, we also executed the same measurement on other platform with different hardware architecture. Although the battery capacity consumption behavior change at a scaling pattern, the relationship between adjusting direction of these parameters and optimal result remained the same as the analyzed conclusion above. The proposed optimization method can be generally repeated on different kind of mobile hardware, the corresponding battery capacity footprinting can be generated, and related parameters changing pattern can also be discovery to provide parameter management guideline for future realtime application on multimedia optimization.

\section{CONCLUSION}

In this work, we builded testbed to measure the battery capacity consumption in multimedia communication and provided a analysis of the proposed optimization framework for wireless video communication systems powered by battery. Based on optimization, the video coding and transmission are jointly optimized to minimize the battery capacity under the required constraint of expected received frame distortion. Experimental results of optimized solutions revealed the relationship between the adjustment of important parameters in both video coding and transmission and the corresponding battery capacity consumption under a given distortion constraint, and this provides an optimal adjusting direction which can be applied in realtime multimedia optimization system without recalculating all the options for coding and transmitting each video frame. The battery capacity consumption footprinting carried out in this work also provides design insights for battery resource allocation in future mobile multimedia systems.

\section{REFERENCES}

[1] Z. He, Y. Liang, L. Chen, I. Ahmad, and D. Wu, "Power-Rate-Distortion Analysis for Wireless Video Communication Under Energy Constraints," vol. 15 , no. 5, pp. 645-658, May 2005.

[2] Z. He and D. Wu, "Resource Allocation and Performance Analysis of Wireless Video Sensors," Circuits and Systems for Video Technology, vol. 15, no. 5, pp. 645-658, May 2005.

[3] A. K. Katsaggelos, F. Zhai, Y. Eisenberg, and R. Berry, "Energyefficient wireless video coding and delivery," IEEE Journal Wireless Communications, vol. 12, pp. 24-30, Aug. 2005.

[4] X. Lu, Y. Wang, and E. Erkip, "Power efficient h.263 video transmission over wireless channels," in International Conference Image Processing, Dec. 2002, pp. I-533-I-536.

[5] D. N. Kwon, P. F. Driessen, A. Basso, and P. Agathoklis, "Performance and computational complexity optimization in configurable hybrid video coding system," vol. 16, no. 1, pp. 31-42, Jan. 2006.

[6] C.-J. Lian, S.-Y. Chien, C.-P. Lin, P.-C. Tseng, and L.-G. Chen, "Poweraware multimedia: Concepts and design perspectives," IEEE Circuits and Systems Magazine, pp. 26-34, 2007.

[7] D. Krishnaswamy and M. van der Schaar, "Adaptive modulated scalable video transmission over wireless networks with a game theoretic approach," in IEEE 6th Multimedia Signal Processing Workshop, Sep. 2004, pp. 107-110.

[8] D. Krishnaswamy, "Game theoretic formulations for network-assisted resource management in wireless networks," in IEEE Vehicular Technology Conference, Dec. 2002, pp. 1312-1316.

[9] - "Network-sddisted link adaptation with power control and channel reassignment in wireless networks," in $3 G$ Wireless Conference, May. 2002, pp. 165-170.

[10] H.264 JM 16.2 reference software [Online]. Available: http://iphome.hhi.de/suehring/tml/download/. 